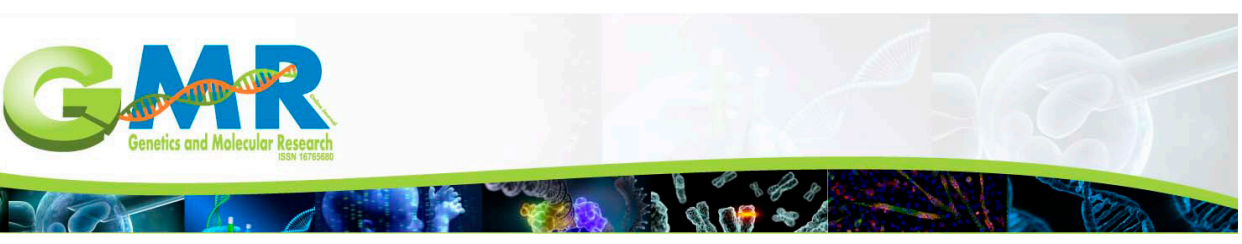

\title{
Molecular cloning and expression vector construction of bovine TRIM28
}

\author{
X. Ma ${ }^{1 *}$, Z.C. Zhai ${ }^{1 *}$, M.L. Zhang1, B.H. Song ${ }^{2}$, Y.R. Zhu ${ }^{1}$, S.B. Yang1, \\ X.Q. Dong ${ }^{1}$, L.Y. Su ${ }^{1}$, C.F. Wang ${ }^{1}$, H.X. Ma $^{1}$ and W.M. Luan ${ }^{1}$ \\ ${ }^{1}$ College of Animal Science and Technology, Jilin Agricultural University, \\ Changchun, Jilin, China \\ ${ }^{2}$ Chang Chun Medical College, Changchun, Jilin, China \\ *These authors contributed equally to this study. \\ Corresponding authors: W.M. Luan / H.X. Ma \\ E-mail: maxin3202@163.com
}

Genet. Mol. Res. 15 (2): gmr.15028793

Received May 16, 2016

Accepted June 3, 2016

Published June 24, 2016

DOI http://dx.doi.org/10.4238/gmr.15028793

\begin{abstract}
The bovine TRIM28 gene was amplified from ovary tissue by using RT-PCR. The TRIM28 gene was inserted into the eukaryotic expression vector pIRES2-EGFP and transfected into bovine fetal fibroblasts by using Lipofectamine 3000. TRIM28 mRNA and protein were detected by fluorescence microscope and western blotting. The results showed that the full length of TRIM28 was cloned and pIRES2-EGFP-TRIM28 was constructed successfully. EGFP expression was observed, and the pIRES2-EGFP-TRIM28 transfected group expressed more TRIM28 protein than that by the pIRES2-EGFP group. The TIMR 28 gene has been successfully transferred into bovine fetal fibroblasts.
\end{abstract}

Key words: TRIM28; pIRES2-EGFP-TRIM28; Transfection 


\section{INTRODUCTION}

Tripartite motif-containing 28 (TRIM28), also known as KRAB domain-associated protein 1 (KAP1) or transcriptional intermediary factor 1 beta (TIF1 $\beta$ ) was discovered by its interaction with the KRAB (Krüppel-associated box) domain found in many transcription factors (Abrink et al., 2001). The KRAB domain is a potent and highly conserved repression domain, existing in about one-third of zinc finger proteins (ZFPs), the largest family of transcription factors. Since TRIM28 binds to the conserved KRAB repression domain, it is considered a critical transcriptional corepressor (Kim et al., 1996; Moosmann et al., 1996; Satou et al., 2001). TRIM28 epigenetically regulates gene expression through multiple transcriptional corepressor complexes. Many proteins form TRIM28-containing complexes, including heterochromatin-associated protein 1 (HP1), histone methyltransferases (HMTs), nuclear corepressor (N-CoR), and histone deacetylases (HDACs) (Gritz and Davies, 1983; Fu et al., 2011; Iyengar et al., 2011; Iyengar and Farnham, 2011). In addition, TRIM28 can also regulate transcription factors lacking KRAB domains, for example, c-Myc and E2F1 (Satou et al., 2001; Satou et al., 2004; Hagio et al., 2006; Ivanov et al., 2007; Wang et al., 2007; Chen et al., 2012). Deletion of TRIM28 in mouse embryo leads to embryonic lethality (Messerschmidt et al., 2012), suggesting that TRIM28 is critical during embryonic development. TRIM28 functions as a scaffold protein to stabilize transcriptional complexes that regulate chromatin remodeling, it also plays an important role in maintaining genome imprint.

TRIM28 has been reported to regulate many aspects of physiology, such as embryonic development, cell differentiation, virus replication, immune response, DNA damage, tumorigenesis, maintenance of genomic integrity, and impairment of learning and memory. These diverse cellular functions of TRIM28 have been showed for several years, but the specific mechanisms TRIM28 utilizes to regulate these various physiology processes are still largely unknown, especially in bovine species. To further investigate the function of TRIM28 in bovine species, it is necessary to clone this gene and construct an expression vector. In this study, the full length bovine TRIM28 was cloned and the expression vector pIRES2-EGFPTRIM28 was constructed successfully. Finally, TRIM28 expression in bovine fibroblasts was observed by fluorescence detection and western blot analysis.

\section{MATERIAL AND METHODS}

\section{Ethics statement}

All animal studies were conducted according to the experimental practices and standards approved by the Animal Welfare and Research Ethics Committee at Jilin Agricultural University.

\section{Total RNA isolation and cDNA synthesis}

Bovine ovaries were collected from a local abattoir and transported to the laboratory at $30^{\circ} \mathrm{C}$ for $2 \mathrm{~h}$. Total ovary RNA was extracted using Trizol Reagent (TaKaRa). To obtain the cDNA template for real time PCR, RNA pellets were dissolved in $\mathrm{DEPC}-\mathrm{H}_{2} \mathrm{O}$ (diethylpyrocarbonatetreated $\mathrm{H}_{2} \mathrm{O}$ ). To remove DNA contamination, 10 units of DNase I (Promega) was added, then cDNA was synthesized using an oligo-dT primer and the SuperScript reverse transcriptase 
performed precisely by the manufacturer instructions (TaKaRa). TRIM28 mRNA expression was detected in bovine ovary by RT-PCR. The primers used were as follows: F: 5'-CATGTGCAACCAGTGCGAAT-3'; R: 5'-TGGGGAGAAGGTGGAGTCAG-3'.

\section{Cloning of bovine TRIM28 open reading frames}

To overcome the long GC-rich TRIM28 sequence, LA Taq DNA polymerase and GC buffer were used. With bovine ovary cDNA as a template, PCR was performed in a final volume of $25 \mu \mathrm{L}$ with the following components: $0.5 \mu \mathrm{L}$ LA Taq DNA polymerase (5 U/ $\mu \mathrm{L}$; TaKaRa), $25 \mu \mathrm{L} 2 \mathrm{X}$ GC Buffer I, $8 \mu \mathrm{L}$ dNTP Mixture ( $2.5 \mathrm{mM}$ each), $2 \mu \mathrm{L}$ TRIM28 cloning primer $(10 \mathrm{mM}), 1 \mu \mathrm{L}$ template cDNA, and $11.5 \mu \mathrm{L} \mathrm{ddH}_{2} \mathrm{O}$. PCR was then performed using the following thermal cycling conditions: $94^{\circ} \mathrm{C}$ for $3 \mathrm{~min}$, followed by 35 cycles of $94^{\circ} \mathrm{C}$ for $30 \mathrm{~s}, 60^{\circ} \mathrm{C}$ for $30 \mathrm{~s}, 72^{\circ} \mathrm{C}$ for $2 \mathrm{~min}$, and a final extension step of $72^{\circ} \mathrm{C}$ for 10 min. The cloning primers were designed according to the Bos taurus TRIM28 mRNA sequence (NCBI Reference Sequence: NM_001206809.1). The cloning primers used were as follows: C-TRIM28-F-5'-GTGAATGGCGGCTTCGGCTGCG-3'; C-TRIM28-R-5'GGAGGAGTGACAGGACATAGA-3'.

The PCR products from the above reaction were combined in a final volume of $50 \mu \mathrm{L}$ with the following reagents: $46.5 \mu \mathrm{L}$ PCR products, $3 \mu \mathrm{L}$ dNTPs, $0.5 \mu \mathrm{L}$ Taq polymerase, then an extension step was performed at $72^{\circ} \mathrm{C}$ for $10 \mathrm{~min}$.

PCR products $(5 \mu \mathrm{L})$ were then separated by electrophoresis on a $0.8 \%$ agarose gel, and bands above $2 \mathrm{~kb}$ were considered positive for TRIM28 amplification.

Positive PCR products were purified (Qiagen, Valencia, CA) and cloned into a PMD18-T vector $(\mathrm{TaKaRa})$. To prepare for transformation, the following reagents were incubated at room temperature for $1 \mathrm{~h}: 4 \mu \mathrm{L}$ of fresh PCR products, $1 \mu \mathrm{L}$ of solution Buffer, and $1 \mu \mathrm{L}$ PMD18-T vector. TOP10 Competent Cells were used for transformation according to the One Shot Chemical Transformation Protocol. To confirm correct size of the DNA inserted, PCR was performed with M13 primers, and samples demonstrating the proper band size were sequenced.

\section{Construction of pIRES2-EGFP-TRIM28 recombinant plasmid}

Using the PMD18-T-TRIM28 vector plasmid as a template, high-fidelity PCR was performed to amplify full length TRIM28 with restriction enzyme digestion sites, and the resulting sequence was cloned into a PMD18-T vector. Primers were designed according to the sequence of the TRIM28 gene and the pIRES2-EGFP multiple cloning sites, SalI and BamHI (both from NEB) restriction enzyme digestion sites. The amplification primers used were as follows: SalI-TRIM28-F: gccgtcgacGTGAATGGCGGCTTCGGCTGCG; BamHITRIM28-R: cgcggatccGGAGGAGTGACAGGACATAGA.

The PMD18-T-TRIM28 plasmids (with restriction enzyme digestion sites added) were digested with SalI and BamHI, and the target fragment, full-length TRIM28 cDNA, was isolated and purified. The eukaryotic expression vector pIRES2-EGFP was also digested with BamHI and SalI, then ligated with TRIM28 cDNA using T4 DNA Ligase (MBI). The transformation of recombinant pIRES2-EGFP-TRIM28 plasmid was performed in TOP10 competent cells. The bacteria containing pIRES2-EGFP-TRIM28 plasmid were processed by a Plasmid Miniprep Kit (TIANGEN). Positive clones were identified by restriction enzyme digestion, PCR amplification, and DNA sequencing. 


\section{Cell transfection}

Bovine fibroblasts were cultured in Dulbecco's Modified Eagle Medium (DMEM) supplemented with $10 \%$ fetal bovine serum, $1 \%$ MEM non-essential amino acids, and $1 \%$ glutamine (all obtained from Gibco) at $37^{\circ} \mathrm{C}$ and $5 \% \mathrm{CO}_{2}$. Before transfection cells were passaged and plated in $24-w e l l$ plates at $70-80 \%$ confluence. Cells were separated into two groups: Lipofectamine $3000+$ pIRES2-EGFP (pIRES2 group), and Lipofectamine3000 + pIRES2-EGFP-TRIM28 (TRIM28 group). Transfection was carried out according to the manufacturer instructions for Lipofectamine 3000. The ratio of Lipofectamine 3000 to pIRES2EGFP-TRIM28 vector was 4:1 (2 $\mu \mathrm{L}: 0.5 \mathrm{mg})$ per well. Before transfection, the mixture was incubated at room temperature for $15 \mathrm{~min}$, then was incubated with cells for $6 \mathrm{~h}$ and medium was changed. Cells were collected $48 \mathrm{~h}$ after transfection for fluorescence observation, and 72 $\mathrm{h}$ after transfection for western blotting analysis.

\section{Western blot assay}

Cells were collected, lysed, and separated by $12 \%$ Glycine-SDS-PAGE. The protein gels were transferred to PVDF (polyvinylidene fluoride) membranes (Invitrogen) in transfer buffer ( $25 \mathrm{mM}$ Tris, $15 \%$ methanol, and $200 \mathrm{mM}$ glycine) using a BioRad semi-dry blotting system at $10 \mathrm{~V}$ for $30 \mathrm{~min}$ to transfer TRIM28 and $10 \mathrm{~V}$ for $20 \mathrm{~min}$ to transfer $\beta$-actin. Membranes were blocked using 5\% skim milk in PBS-T (PBS containing 0.1\% Tween 20) for $2 \mathrm{~h}$ with gentle agitation, and washed in PBS (3 times, 10 min each). Blots were probed with a primary antibody toward bovine TRIM28 (SC-33186, Santa Cruz Biotechnology, Inc.) or $\beta$-actin (bs-0061R, Bioss, china) at $4^{\circ} \mathrm{C}$ overnight. Membranes were then incubated with secondary antibody conjugated a HRP (10 min) at room temperature and washed in PBS (3 times, 10 min each). Protein bands were showed with ECL Plus.

\section{RESULTS}

\section{Expression of TRIM28 mRNA in bovine ovary}

TRIM28 is essential for normal development and differentiation. Recent research reported that TRIM28 is expressed in murine ovaries and oocytes and various cancer cell lines, but is not expressed in normal tissues (Messerschmidt et al., 2012). TRIM28 mRNA was detected in bovine ovary by RT-PCR. Our results showed that TRIM28 mRNA was detected in bovine ovary by RT-PCR, and therefore, is expressed in bovine ovary (Figure 1).

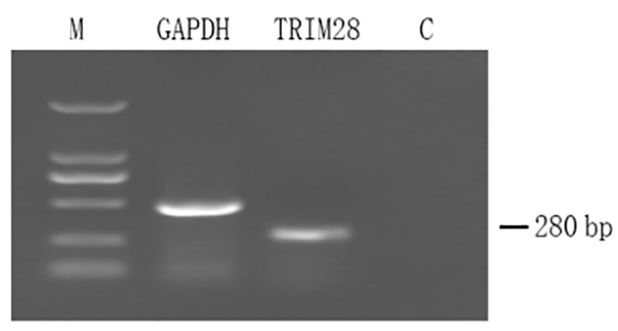

Figure 1. TRIM28 mRNA expressed in bovine ovary. Lane $M=$ DL2000; lane $C$ = water control. 


\section{Cloning of bovine TRIM28}

With bovine ovary cDNA as a template, LA Taq DNA polymerase amplification of the TRIM 28 gene was performed by RT-PCR. The RT-PCR products were analyzed by $0.8 \%$ agarose gels, and the full-length TRIM28 cDNA band was observed at $2572 \mathrm{bp}$ (Figure 2). The bovine TRIM28 clone is 2572 bp in length with the start codon found at nucleotide 5, and the TGA stop codon located at nucleotide $2433 \mathrm{bp}$. The ORF of bovine TRIM28 is 2438 bp, shows a GC content of 59.4\%, and encodes a protein containing 812 amino acids. The TRIM28 polypeptide has an estimated molecular mass of $88.6 \mathrm{kDa}$. The PCR products were cloned into a PMD18-T vector. The RT-PCR products were confirmed 100\% homology with the bovine TRIM28 mRNA (NM_001206809.1) by direct sequencing.

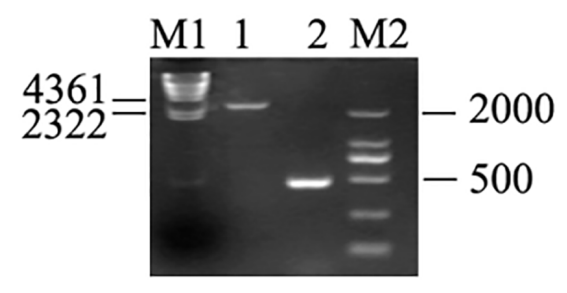

Figure 2. Full-length bovine TRIM 28 cDNA obtained from ovary mRNA using RT-PCR. Lane M1 = $\lambda$ DNA; lane $M 2=$ DL2000; lane $1=$ RIM28; lane $2=\mathrm{GAPDH}$.

The TRIM28 coding sequence was compared to the human, pig, and mouse (Table 1) sequences. Bovine TRIM 28 shared 88,91 , and $83 \%$ nucleotide sequence homology to human, pig, and mouse sequences, respectively. A multiple alignment of bovine TRIM28 with other TRIM28 proteins, shown in Table 1 and Figure 3, demonstrated very high sequence homology. The bovine TRIM28 amino acid homology with human, pig, and mouse proteins was 97, 97, and $94 \%$, respectively.

Table 1. Homology of bovine TRIM28 coding sequence.
\begin{tabular}{l|c|c}
\hline & Nucleotide (\%) & Amino acid (\%) \\
\hline Length of bovine coding sequence & 2438 & 812 \\
\hline Identity against human & 88 & 97 \\
\hline Identity against Porcine (Hamm et al., 2014) & 91 & 97 \\
\hline Identity against mouse & 83 & 94 \\
\hline
\end{tabular}

\section{Construction of pIRES2-EGFP-TRIM28 vector and transfecting bovine fibroblasts}

In order to add restriction enzyme digestion sites (SalI and BamHI) to the cDNA, high fidelity PCR polymerase amplification of the TRIM28 gene with restriction enzyme digestion sites was performed with PMD18-T-TRIM28 as a template. Then the expression vector pIRES2-EGFP and TRIM28 cDNA with restriction enzyme digestion sites were doubly digested with SalI and BamHI. Then pIRES2-EGFP and TRIM28 were ligated. Positive clones were identified by restriction enzyme digestion (Figure 4), PCR analysis, and DNA sequencing. The expression vector pIRES2-EGFP-TRIM28 was transfected into bovine fetal fibroblasts using Lipofectamine 3000. Green fluorescence was observed in about 30\% 
of bovine fibroblasts $48 \mathrm{~h}$ after transfection (Figure 5). Protein expression of TRIM28 was determined by western blotting $72 \mathrm{~h}$ after transfection. Results showed that the TRIM28expressing group expressed more TRIM28 protein than the pIRES2 group (Figure 6).

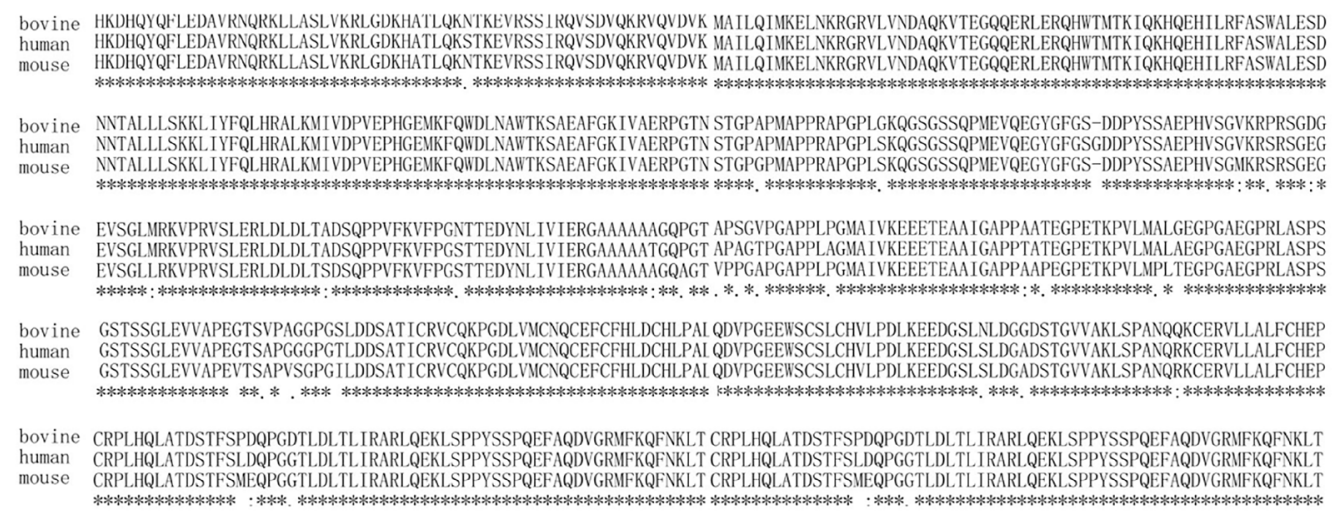

Figure 3. ClustalX alignment of the bovine TRIM28 protein sequence with human and mouse sequences. *Conserved amino acid residues, dots $=$ high similarity, colons $=$ low similarity.

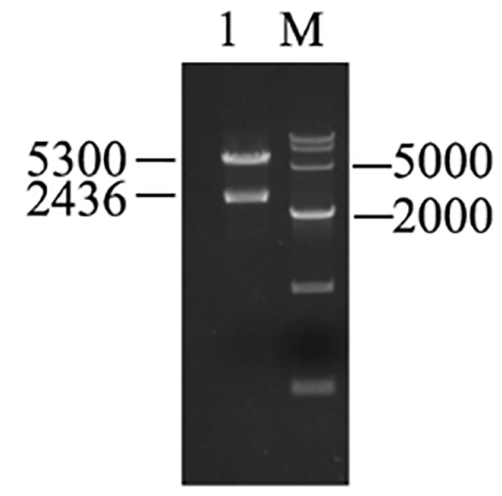

Figure 4. BamHI and SalI digestion and electrophoresis of pIRES2-EGFP-TRIM28. Lane $M=$ DL15,000 DNA marker; lane 1 = pIRES2-EGFP-TRIM28 expression vector digested with SalI and BamHI.

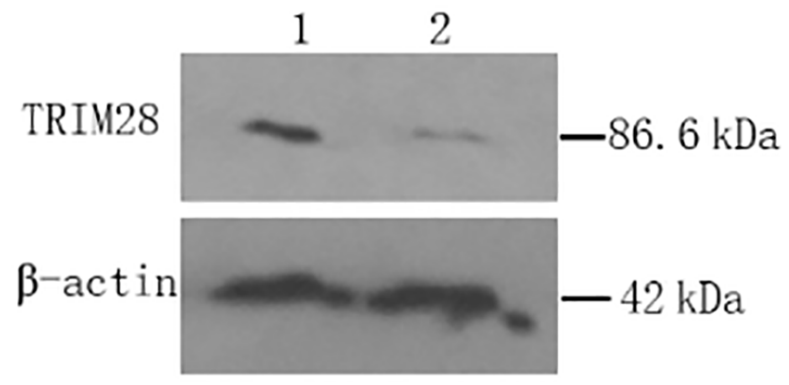

Figure 5. Green fluorescence in bovine fibroblasts transfected with pIRES2-EGFP-TRIM28. A. Fluorescence image (570X). B. Phase contrast image (570X). 


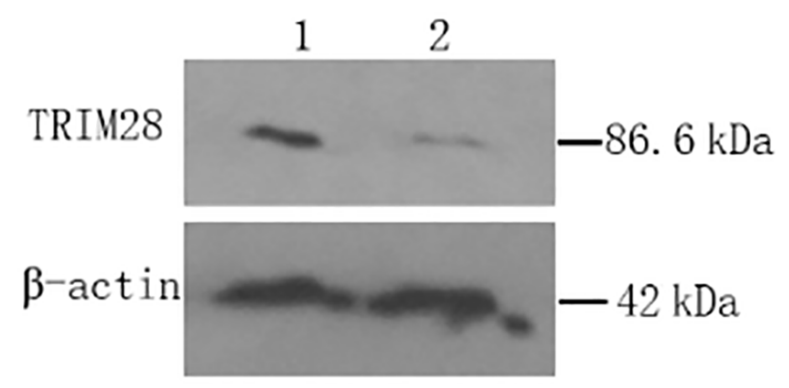

Figure 6. TRIM28 group expressed more TRIM28 protein than pIRES2 group. Lane $1=$ TRIM28 group; lane $2=$ pIRES2 group.

\section{DISCUSSION}

TRIM28 plays an important role in multiple cellular processes, and is widely expressed in different cells (with especially high expression in some cancer lines). However, the expression level of TRIM28 varies in different cell types, and no comprehensive study of TRIM28 expression has yet been published. Therefore, the establishment of an efficient TRIM28 expression vector is necessary to facilitate these studies. In the present study, bovine TRIM28 was cloned; the full-length TRIM28 cDNA is located on chromosome 18 and contained a 2,438 nucleotide coding sequence (CDS) encoding a polypeptide of 812 amino acids with molecular weight of $86.6 \mathrm{kDa}$. Many researches have revealed that TRIM28 is SUMOylated and the SUMOylation of TRIM28 is needed for its transcriptional repressive function (Lee et al., 2007; Li et al., 2007; Mascle et al., 2007), The GC content of the 5'-end $(1-1580 \mathrm{bp}$ ) reaches $62.6 \%$, so it is very difficult to design primers and amplify the full length sequence of TRIM28. We designed several primer pairs to clone it, and also to overcome the long and GC-rich sequence, LA Taq DNA polymerase and GC buffer were used. Finally the long GC-rich TRIM28 was cloned successfully.

Lipofectamine 3000 is a proprietary formulation for transfecting nucleic acids into a wide range of eukaryotic cells. Lipofectamine 3000 transfection reagent has fewer negative effects, greater repeatability, and easy to transfect compared to other reagents. Therefore, we chose this reagent for our studies. The expression vector pIRES2-EGFP has neomycin and kanamycin double resistance genes for eukaryotic and prokaryotic selection, and an enhanced green fluorescent protein (EGFP) gene as reporter gene. This vector also has an Internal Ribosome Entry Site (IRES); when an IRES segment is located between two reporter open reading frames in a eukaryotic mRNA molecule, it can drive translation of the downstream protein coding region independently of the 5 '-cap structure bound to the 5 ' end of the mRNA molecule. In such a vector, both proteins are produced in the cell, so the inserted gene (TRIM28 in this case) and the reporter gene EGFP express simultaneously. EGFP facilitates the observation and selecting of positive cells under a fluorescence microscope. Green fluorescence in cells was observed in TRIM28 group, which indicated the EGFP genes had been transfected into bovine fibroblasts successfully. PIRES2-EGFPTRIM28 was constructed and identified by RT-PCR and western blotting. The results showed that bovine fibroblasts transfected with pIRES2-EGFP-TRIM28 expressed more TRIM28 than cells transfected with the empty vector, which indicates that TRIM28 was transferred into bovine fibroblasts successfully. 
In conclusion, full length TRIM28 was cloned and the eukaryotic expression vector pIRES2-EGFP-TRIM28 was constructed successfully. The bovine fibroblasts which expressed the TRIM28 gene and protein were transfected using the liposome method. The most widely used methodology for functional studies of target genes involve artificial overexpression or knockdown the target gene by applying molecular technologies. So TRIM28 molecular cloning and overexpression vector pIRES2-EGFP-TRIM28 might prove useful for studies of its function.

\section{ACKNOWLEDGMENTS}

Research supported by grants from National Natural Science Foundation (\#31302047) and the University Students Innovation and Business Startups Projects (\#201510193021) in China.

\section{REFERENCES}

Abrink M, Ortiz JA, Mark C, Sanchez C, et al. (2001). Conserved interaction between distinct Krüppel-associated box domains and the transcriptional intermediary factor 1 beta. Proc. Natl. Acad. Sci. USA 98: 1422-1426.

Chen L, Chen DT, Kurtyka C, Rawal B, et al. (2012). Tripartite motif containing 28 (Trim28) can regulate cell proliferation by bridging HDAC1/E2F interactions. J. Biol. Chem. 287: 40106-40118. http://dx.doi.org/10.1074/jbc.M112.380865

Fu J, Qin L, He T, Qin J, et al. (2011). The TWIST/Mi2/NuRD protein complex and its essential role in cancer metastasis. Cell Res. 21: 275-289. http://dx.doi.org/10.1038/cr.2010.118

Gritz L and Davies J (1983). Plasmid-encoded hygromycin B resistance: the sequence of hygromycin B phosphotransferase gene and its expression in Escherichia coli and Saccharomyces cerevisiae. Gene 25: 179-188. http://dx.doi. org $/ 10.1016 / 0378-1119(83) 90223-8$

Hagio Y, Kimura Y, Taira T, Fujioka Y, et al. (2006). Distinct localizations and repression activities of MM-1 isoforms toward c-Myc. J. Cell. Biochem. 97: 145-155. http://dx.doi.org/10.1002/jcb.20619

Hamm J, Tessanne K, Murphy CN and Prather RS (2014). Transcriptional regulators TRIM28, SETDB1, and TP53 are aberrantly expressed in porcine embryos produced by in vitro fertilization in comparison to in vivo- and somatic-cell nuclear transfer-derived embryos. Mol. Rep. Dev. 81: 552-566.

Ivanov AV, Peng H, Yurchenko V, Yap KL, et al. (2007). PHD domain-mediated E3 ligase activity directs intramolecular sumoylation of an adjacent bromodomain required for gene silencing. Mol. Cell 28: 823-837. http://dx.doi. org/10.1016/j.molcel.2007.11.012

Iyengar S and Farnham PJ (2011). KAP1 protein: an enigmatic master regulator of the genome. J. Biol. Chem. 286: 2626726276. http://dx.doi.org/10.1074/jbc.R111.252569

Iyengar S, Ivanov AV, Jin VX, Rauscher FJ, 3rd, et al. (2011). Functional analysis of KAP1 genomic recruitment. Mol. Cell. Biol. 31: 1833-1847. http://dx.doi.org/10.1128/MCB.01331-10

Kim SS, Chen YM, O'Leary E, Witzgall R, et al. (1996). A novel member of the RING finger family, KRIP-1, associates with the KRAB-A transcriptional repressor domain of zinc finger proteins. Proc. Natl. Acad. Sci. USA 93: 1529915304.http://dx.doi.org/10.1073/pnas.93.26.15299

Lee YK, Thomas SN, Yang AJ and Ann DK (2007). Doxorubicin down-regulates Kruppel-associated box domainassociated protein 1 sumoylation that relieves its transcription repression on $\mathrm{p} 21 \mathrm{WAF} 1 / \mathrm{CIP} 1$ in breast cancer MCF-7 cells. J. Biol. Chem. 282: 1595-1606. http://dx.doi.org/10.1074/jbc.M606306200

Li X, Lee YK, Jeng JC, Yen Y, et al. (2007). Role for KAP1 serine 824 phosphorylation and sumoylation/desumoylation switch in regulating KAP1-mediated transcriptional repression. J. Biol. Chem. 282: 36177-36189. http://dx.doi. org/10.1074/jbc.M706912200

Mascle XH, Germain-Desprez D, Huynh P, Estephan P, et al. (2007). Sumoylation of the transcriptional intermediary factor 1 beta (TIF1beta), the Co-repressor of the KRAB Multifinger proteins, is required for its transcriptional activity and is modulated by the KRAB domain. J. Biol. Chem. 282: 10190-10202. http://dx.doi.org/10.1074/jbc.M611429200

Messerschmidt DM, de Vries W, Ito M, Solter D, et al. (2012). Trim28 is required for epigenetic stability during mouse oocyte to embryo transition. Science 335: 1499-1502. http://dx.doi.org/10.1126/science.1216154

Moosmann P, Georgiev O, Le Douarin B, Bourquin JP, et al. (1996). Transcriptional repression by RING finger protein 
TIF1 beta that interacts with the KRAB repressor domain of KOX1. Nucleic Acids Res. 24: 4859-4867. http://dx.doi. org $/ 10.1093 / \mathrm{nar} / 24.24 .4859$

Satou A, Taira T, Iguchi-Ariga SM and Ariga H (2001). A novel transrepression pathway of c-Myc. Recruitment of a transcriptional corepressor complex to c-Myc by MM-1, a c-Myc-binding protein. J. Biol. Chem. 276: 46562-46567. http://dx.doi.org/10.1074/jbc.M104937200

Satou A, Hagio Y, Taira T, Iguchi-Ariga SM, et al. (2004). Repression of the c-fms gene in fibroblast cells by c-Myc-MM1-TIF1beta complex. FEBS Lett. 572: 211-215.http://dx.doi.org/10.1016/j.febslet.2004.07.034

Wang C, Rauscher FJ, 3rd, Cress WD and Chen J (2007). Regulation of E2F1 function by the nuclear corepressor KAP1. J. Biol. Chem. 282: 29902-29909. http://dx.doi.org/10.1074/jbc.M704757200 